\title{
Monte Carlo Study on the Water Meniscus Condensation and Capillary Force in Atomic Force Microscopy
}

\author{
Hyojeong Kim, ${ }^{\dagger}$ Berend Smit, ${ }^{\dagger}$ and Joonkyung Jang ${ }^{* \dagger}$ \\ ${ }^{\dagger}$ Department of Nanomaterials Engineering, Pusan National University, Miryang 627-706, Republic of Korea \\ ${ }^{\ddagger}$ Department of Chemistry and Department of Chemical and Biomolecular Engineering, University of California, Berkeley, California \\ 94720-1460, United States
}

ABSTRACT: The water meniscus condensed between a nanoscale tip and an atomically flat gold surface was examined under humid conditions using grand canonical Monte Carlo simulations. The molecular structure of the meniscus was investigated with particular focus on its width and stability.

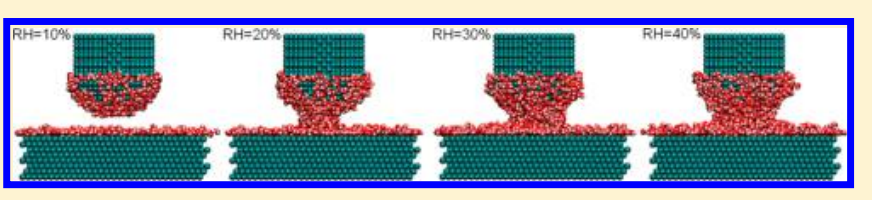
The capillary force due to the meniscus showed a dampened oscillation with increasing separation between the tip and surface because of the formation and destruction of water layers. The layering of water between the tip and the surface was different from that of the water confined between two plates. The humidity dependence of the capillary force exhibited a crossover behavior with increasing humidity, which is in agreement with the typical atomic force microscopy experiment on a hydrophilic surface.

\section{INTRODUCTION}

An atomic force microscope (AFM) tip with an apex on the nanometer scale is used widely for imaging and molecular patterning of surfaces. ${ }^{1,2}$ In these processes, the ambient water vapor condenses spontaneously into a liquid meniscus, which bridges the tip and surface., ${ }^{3,4}$ This phenomenon is a manifestation of capillary condensation caused by the confinement of vapor trapped between two solids (tip and surface). ${ }^{5,6}$ The nascent meniscus exerts an adhesion force (typically, several $\mathrm{nN}$ in magnitude), which must be supplied to retract the tip from the surface. This capillary force dominates over the van der Waals and electrostatic forces between the tip and surface. Therefore, it is important to understand the capillary force to interpret the AFM images under ambient conditions. In dip pen nanolithography (DPN), ${ }^{7}$ the meniscus mediates the transfer of molecules initially coated on the tip down to the surface, where molecular patterns are deposited. Here, the shape and size of the meniscus play vital roles in controlling the outcome of DPN. ${ }^{8}$

Despite its wide occurrence in AFM and DPN experiments, the current understanding of the water meniscus is incomplete, particularly at the molecular level. Because it is difficult to visualize a nanometer-sized meniscus, ${ }^{9,10}$ what is typically observed in the experiment is the force exerted on the tip. ${ }^{11,12}$ The meniscus structure (e.g., its width and curvature) is often deduced from the venerable Kelvin equation: ${ }^{13-17}$

$$
R T \ln \left(p / p_{0}\right)=\gamma V\left(\frac{1}{r_{1}}+\frac{1}{r_{2}}\right)
$$

where $p$ is the pressure, $p_{0}$ is the saturation pressure of water vapor, $T$ is the temperature, $\gamma$ is the liquid-vapor surface tension, and $V$ is the molar volume of liquid water. $r_{2}$ in eq 1 is the neck radius of the meniscus (here, the meniscus is assumed to have a cylindrical symmetry around the tip axis), and $r_{1}$ is the radius of the curvature of the meniscus periphery. $r_{1}$ is negative for a concave meniscus but positive for a convex one. Kelvin theory has been elaborated by considering a nonspherical tip shape, the existence of a water film, ${ }^{18}$ and the effects of liquid evaporation. ${ }^{19}$ On the other hand, Xiao and Qian ${ }^{18}$ concluded that continuum theory cannot reproduce the experimental behavior of the pull-off force with respect to humidity. More importantly, the Kelvin equation, based on a continuum picture of water, has inherent limitations in delivering the discrete molecular insights on the meniscus.

Molecular simulations have filled in the gaps of our knowledge about the molecular details on the meniscus. By using a molecular dynamics (MD) simulation, the contact angle of a water droplet interacting with a probe tip was studied. ${ }^{20} \mathrm{~A}$ $\mathrm{MD}$ simulation $^{21-23}$ was used to examine the formation, thinning, and snap-off of the meniscus as an AFM tip retracts from a surface. The periphery of a nanometer-sized meniscus fluctuated significantly. ${ }^{22}$ By using the meniscus structure obtained from a MD simulation, the capillary force ${ }^{24}$ on the tip was calculated using the Kelvin equation. ${ }^{21}$ Note that typical AFM and DPN experiments are performed under ambient conditions. The meniscus is characterized as an open system in equilibrium with the surrounding vapor. This situation is therefore represented most closely by the grand canonical (GC) ensemble. Jang et al. $^{25-27}$ studied the meniscus and capillary force in a series of GC Monte Carlo (GCMC) simulations based on the lattice gas model of water. ${ }^{23}$ Semianalytical density functional theory was also used to examine the meniscus structure $^{28}$ and capillary force. ${ }^{29}$ However, the lattice gas model is highly coarse grained and based on the nearest neighbor interactions between molecules. The model does not consider the orientation of water

Received: August 7, 2012

Revised: September 19, 2012

Published: September 21, 2012 


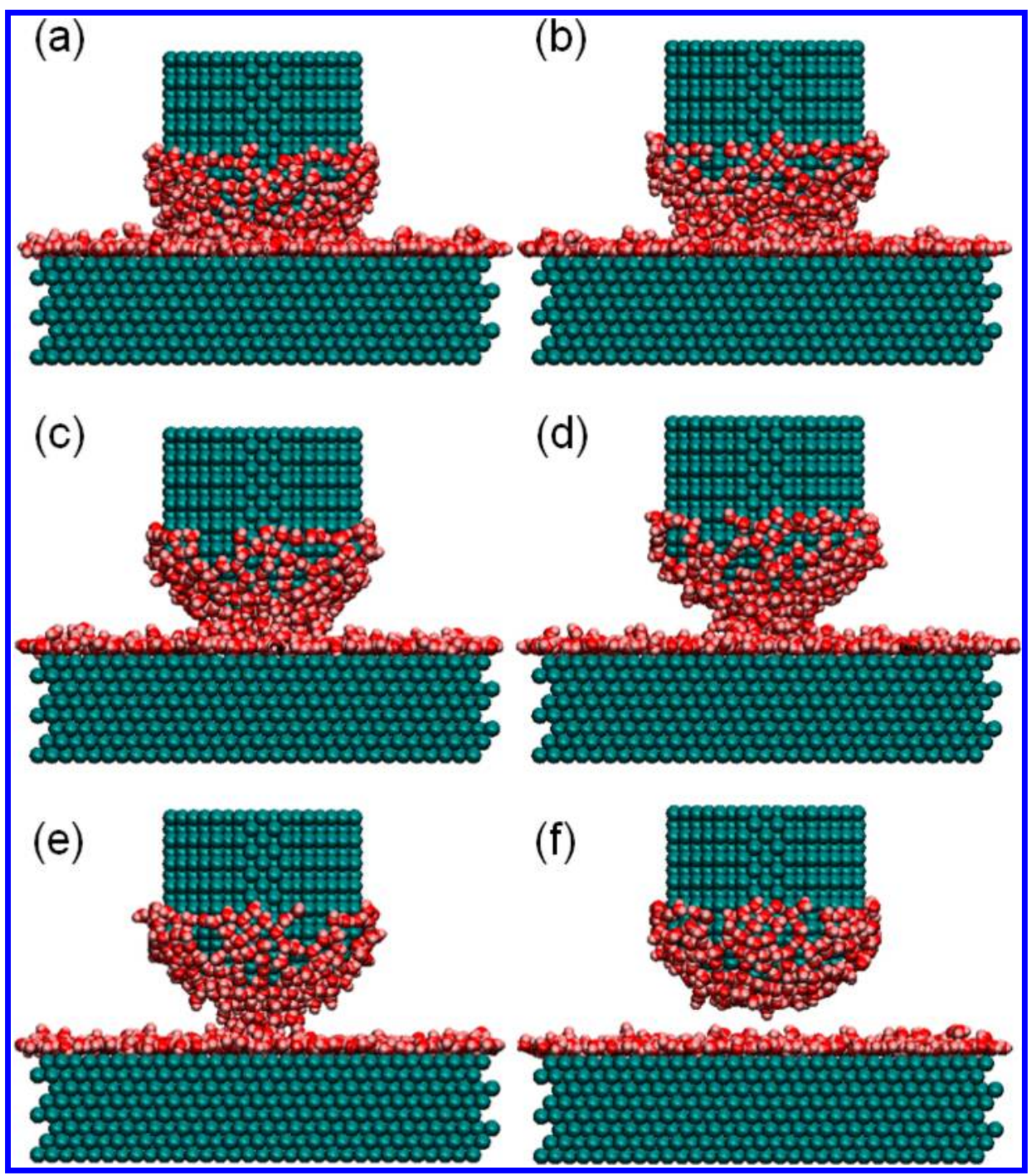

Figure 1. Structural change in the meniscus as the tip is retracted from the surface. Shown are representative configurations of the water meniscus condensed between the tip and flat surface both made from gold atoms. Water molecules above the hemispherical part of the tip are not drawn. The tip-surface separation $d$ was varied as 6 (a), 8 (b), 10 (c), 12 (d), 13 (e), and $14 \AA$ (f).

molecules and the long-range electrostatic interactions between molecules. Therefore, fully atomistic insights, such as the tetrahedral hydrogen-bond network and the alignment of electric dipoles of water on the surface, ${ }^{30-32}$ cannot be captured in the lattice model.

This study investigated the water meniscus confined between an AFM tip and a flat gold surface using a fully atomistic GCMC simulation. The present system serves as a prototype of an AFM experiment for an atomically flat and hydrophilic surface. A gold surface was chosen because of its frequent use in humidity dependent AFM experiments ${ }^{9,10,33}$ and DPN. ${ }^{1,8}$ The molecular details of the meniscus structure were examined by systematically varying both the humidity and separation between the tip and surface. The width and height of the meniscus and its stability were analyzed. The capillary force resulting from the meniscus was calculated. The capillary force shows a damped oscillation with respect to the tip-surface separation. This behavior originates from the complete and incomplete layering of water between the tip and surface. The pull-off force versus humidity showed crossover (increasing and then decreasing) behavior with increasing humidity, which was in agreement with the AFM experiments of a hydrophilic tip in contact with a hydrophilic surface.

\section{SIMULATION METHOD}

A gold tip positioned above a gold (111) surface was simulated. The gold surface was modeled as a $17 \AA$ thick slab made of eight atomic layers with a lateral (along the $x$ and $y$ axes) size of $81 \AA \times 81 \AA$. The lattice spacing $(2.88 \AA)$ of gold (facecentered cubic) was taken from the literature. ${ }^{34}$ The tip was generated as follows. A simple cubic lattice was first constructed with a spacing of the Lennard-Jones (LJ) $\sigma$ value of gold, and the lattice points located inside a sphere, $2 \mathrm{~nm}$ in radius, was then selected. The bottom half of the sphere was taken, and seven layers of $\mathrm{Au}$ atoms were added to the top of the hemisphere in the form of a cylinder (see Figure 1). The tip and surface were made from 1692 and 7168 atoms, respectively.

The simple point charge (SPC) model of water was used. The model treats each water molecule as three point charges rigidly bound together $(-0.82$ and +0.41 on oxygen and hydrogen atoms, respectively). ${ }^{35}$ The partial atomic charges interact through the Coulomb potential: 


$$
U^{\mathrm{C}}\left(r_{i j}\right)=\frac{q_{i} q_{j}}{4 \pi \varepsilon_{0} r_{i j}}
$$

where $q_{i}$ is the charge on atom $i$ (oxygen or hydrogen) and $\varepsilon_{0}$ is the vacuum permittivity. $r_{i j}$ is the distance between two atoms $i$ and $j$. Oxygen atoms interacted with themselves through the pairwise LJ potential: ${ }^{36}$

$$
U^{\mathrm{LJ}}\left(r_{i j}\right)=4 \varepsilon_{\mathrm{OO}}\left[\left(\frac{\sigma_{\mathrm{OO}}}{r_{i j}}\right)^{12}-\left(\frac{\sigma_{\mathrm{OO}}}{r_{i j}}\right)^{6}\right]
$$

where $\varepsilon_{\mathrm{OO}}$ and $\sigma_{\mathrm{OO}}$ are the LJ energy and length parameters, respectively. Oxygen atoms also interact with gold atoms (of the tip and surface) via the LJ interaction. The LJ parameters $\varepsilon_{i j}$ and $\sigma_{i j}$ for the interaction between different atomic species $i$ (with $\varepsilon_{i i}$ and $\sigma_{i i}$ ) and $j$ (with $\varepsilon_{j j}$ and $\sigma_{j j}$ ) were calculated using the Lorentz-Berthelot mixing rule: ${ }^{36}$

$$
\varepsilon_{i j}=\sqrt{\varepsilon_{i i} \varepsilon_{j j}} \text { and } \sigma_{i j}=\left(\sigma_{i i}+\sigma_{j j}\right) / 2
$$

The LJ parameters of oxygen and gold atoms were $3.166 \AA$ and $0.650 \mathrm{~kJ} / \mathrm{mol}^{35}$ and $2.629 \AA$ and $22.1375 \mathrm{~kJ} / \mathrm{mol}^{37}$ respectively.

The GCMC simulations were run by fixing the temperature, volume, and fugacity. By using the Peng-Robinson equation of state for water, ${ }^{38}$ the fugacity was converted to pressure $p$. The temperature was fixed at $298 \mathrm{~K}$, and the bulk saturation pressure of water, $p_{0}$, was taken as the experimental value, 3167 $\mathrm{Pa}^{39} \mathrm{~A}$ series of simulations were run by changing the relative humidity $(\mathrm{RH}), p / p_{0}$, from $5 \%$ to $70 \%$. The vertical separation of the tip from the surface $d$ was varied from 5 to $20 \AA$ (with an increment of $1 \AA$ ).

Three types of GCMC moves were carried out: translation and rotation of a single molecule, and an exchange of a molecule with one (either real or ghost) in the reservoir. The gold atoms of the tip and surface were frozen in the simulation. A cutoff radius of $16.5 \AA$ was applied to all LJ interactions. The long-range electrostatic interactions were handled using the Ewald-summation technique. ${ }^{36,40}$ Periodic boundary conditions were applied. ${ }^{40}$ Each simulation was equilibrated for at least $10^{6}$ $\mathrm{MC}$ steps. After equilibration, $10^{6} \mathrm{MC}$ steps productions runs were carried out, and MC snapshots are sampled every 100 steps to calculate the ensemble-averaged properties. After equilibration, GCMC run was divided into five blocks, and the average number of molecules in each block was calculated. The number of water molecules does not change much from block to block, and the standard deviation of the number was within $5 \%$, as shown in the standard GCMC simulations. ${ }^{41}$ A detailed description of the present GCMC simulation methods can be found elsewhere. ${ }^{42}$

By using the equilibrium configurations generated in the GCMC simulation, the density profile of water $\rho$ was calculated as follows. By assuming cylindrical symmetry of the meniscus around the tip axis (along the $\mathrm{Z}$ axis), $\rho$ was taken as a function of the vertical height from the surface, $h$, and the lateral distance from the tip axis $r, \rho(r, h) . h$ and $r$ were binned to obtain $\rho(r, h)$ by averaging over the configurations: the bin size for $h$ was $3 \AA$. The radial bin boundaries were located at $r_{i}=(i \delta A / \pi)^{1 / 2}$, where $i=1,2,3, \ldots$, and $\delta A$ is the base area per radial bin, $95 \AA^{2}{ }^{43}$ This way, each bin of $\rho$ has an equal volume (isochore binning). Only oxygen atoms were included when calculating $\rho$, and 1000 configurations were used for averaging. The density profile of atomic charge $\rho_{\mathrm{e}}$ was also checked by averaging over the partial charges of atoms following the same method mentioned above. To extract the structural parameters of the meniscus from $\rho$, the horizontal line in $\rho(r, h)$ was drawn at the middle point between the tip and surface, $h=d / 2$. This line, $\rho(r, h=d / 2)$, behaves like a rounded step function of $r$, whose value decreases from a liquid density $\rho_{1}$ to a vapor density $\rho_{\mathrm{v}}$. The meniscus width at its neck, $2 r_{2}$, was determined by fitting $\rho(r, h=d / 2)$ to the following function: ${ }^{36}$

$$
\begin{aligned}
\rho(r, h & =d / 2) \\
& =\frac{1}{2}\left(\rho_{1}+\rho_{\mathrm{v}}\right)-\frac{1}{2}\left(\rho_{1}-\rho_{\mathrm{v}}\right) \tanh \left[\frac{2\left(r-r_{2}\right)}{d_{\mathrm{s}}}\right]
\end{aligned}
$$

where $d_{s}$ is the thickness of the meniscus periphery, which represents the fluctuations in the periphery. The LevenbergMarquardt nonlinear fitting method ${ }^{44}$ was used to determine $\rho_{\mathrm{v}}$, $\rho_{\mathrm{v}}, d_{\mathrm{s}}$, and $r_{2}$.

The force on the tip $\vec{F}$ was calculated as $\vec{F}=-\nabla U$, where $U$ is the interatomic potentials summed over the tip atoms. By partitioning $U$ into the tip-surface and the tip-water interaction potentials, $\vec{F}$ is projected into the van der Waals force from the surface and capillary force due to the meniscus. The average force exerted on the tip was calculated by averaging $\vec{F}$ over 1000 GCMC configurations. This gives us the average force along the vertical direction, $F_{z}$, with negligible force components along the lateral direction (along the $x$ and $y$ axes).

A MD simulation of two parallel gold (111) plates immersed in water (a slit system) was run for comparison. The layering of water confined between the plates was examined by changing the distance between the two plates. Each plate is a gold (111) surface, which has lateral dimensions of $40 \AA \times 40.32 \AA$ and consists of 1344 gold atoms. The thickness of the plate was 11.8 $\AA$ and was made of six gold layers. The slit was filled with liquid water at room temperature. The number of water molecules was varied from 141 to 410 as the plate-to-plate distance changed from 5 to $10 \AA$. A constant temperature (NVT) MD simulation was run at $298 \mathrm{~K}$ using the Berendsen thermostat. ${ }^{45}$ The MD trajectory was propagated using the velocity Verlet algorithm ${ }^{40}$ with a time step of $1 \mathrm{fs}$. The gold atoms were held rigid in the simulation. The whole simulation time was $1 \mathrm{~ns}$. The DLPOLY ${ }^{46}$ package was used to implement the MD methods described above. By averaging over $1000 \mathrm{MD}$ snapshots, the charge density of water molecules $\rho_{\mathrm{e}}$ for the slit system was calculated.

\section{RESULTS AND DISCUSSION}

The meniscus structure was examined by changing the vertical separation of the tip end from the surface top $d$. Figure 1 presents the representative GCMC configurations for $d$ values of 6 (a), 8 (b), 10 (c), 12 (d), 13 (e), and $14 \AA$ (f), all taken at a $\mathrm{RH}$ of $10 \%$. Water molecules lying above the hemispherical part of the tip were not drawn in the figure. As the tip was retracted from the surface, the meniscus decreased in width but increased in height, and the concave nature of the meniscus became more distinct. At $d=14 \AA$ (Figure 1f), the meniscus finally snapped off. Regardless of $d$, the gold surface was covered completely with a monolayer of water with some water molecules on top of the monolayer. In the range of RHs considered in the present simulation (5-70\%), the second layer was only partially covered with molecules. The mean thickness of the water layers ranged from 3.5 to $6.5 \AA$. The tip was covered only partially 
with water, except near the tip end where the maximal confinement gives full coverage.

The density profile of water $\rho(r, h)$ was calculated by averaging over the GCMC snapshots, as shown in Figure 1. Figure $2 \mathrm{a}-\mathrm{d}$ shows $\rho(r, h)$ for $d$ values of $6(\mathrm{a}), 8(\mathrm{~b}), 10$ (c),

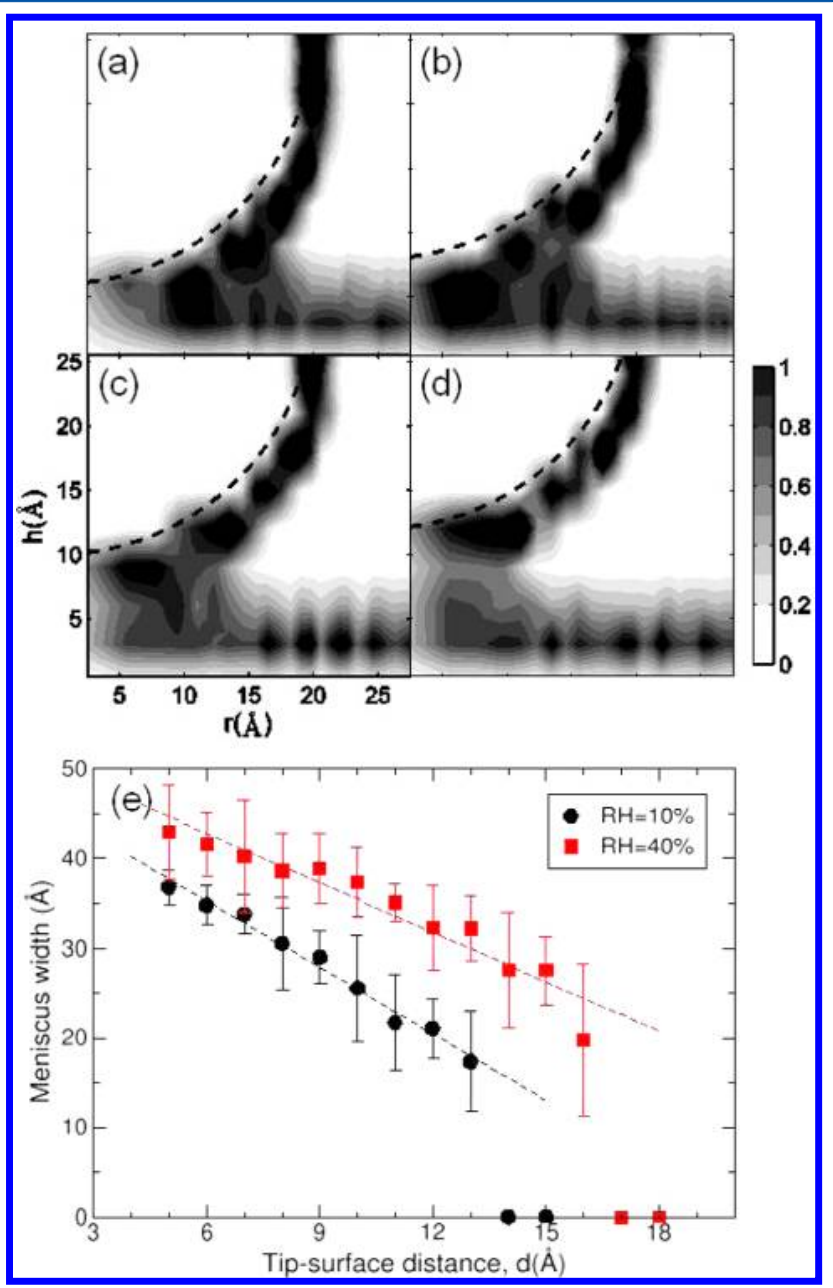

Figure 2. $(a-d)$ Density profile of the water meniscus. The density profile of water is contour plotted as a function of the lateral distance from the tip axis, $r$, and the vertical distance from the surface, $h$. The tip-surface separation $d$ was varied as 6 (a), 8 (b), 10 (c), and $12 \AA$ (d). In the figure, the relative humidity was taken to be $10 \%$. In each panel, the tip boundary was drawn as a broken line. The surface is located at $h=0$. The horizontal and vertical axis scales drawn in (c) apply to (a), (b), and (d). (e) The meniscus width vs the tip-surface separation $d$ for a relative humidity of $10 \%$ (circles) and $40 \%$ (squares). The lines are linear fits to the data, and the error bars represent the thickness of the meniscus periphery $d_{s}$.

and $12 \AA(\mathrm{d})$ at $\mathrm{RH}=10 \% . \rho(r, h)$ decreased from $1.0 \mathrm{~g} / \mathrm{cm}^{3}$ to zero as the lateral distance from the tip axis, $r$, was increased from zero. This was attributed to a gradual decrease in the degree of confinement between the tip and surface as the lateral distance $r$ was increased from zero. On the other hand, $\rho(r, h)$ was not necessarily maximal along the tip axis where the confinement was greatest. The maximum of $\rho(r, h)$ for the shortest $d, 6 \AA$ (Figure 2a), is actually located slightly off axis. This is because two layers of water cannot fit in along the tip axis, $r=0$, (see below for more information on the layering of water between the tip and surface). Similarly, in the case of a tip in direct contact with the surface $(d \leq 3 \AA)$, the meniscus is an annular ring when viewed along the tip axis due to the squeezing out of water from the contact region. ${ }^{22}$

The density profiles (as shown in Figure $2 a-d$ ) are used further to extract the structural parameters of the meniscus, such as its width, by following the fitting procedure described in the Simulation Method section. As in the previous lattice gas $\mathrm{GCMC}^{47}$ and $\mathrm{MD}^{22}$ simulations, $\rho(r, h)$ above (below) half of the bulk liquid density, $0.5 \mathrm{~g} / \mathrm{cm}^{3}$, was taken to be in the liquid (vapor) phase. The meniscus width, $2 r_{2}$, was obtained by varying $d$ from 5 to $15 \AA$ (Figure $2 \mathrm{e}$ ). Drawn as lines in Figure $2 \mathrm{e}$ are the linear fit to the meniscus width versus the tipsurface distance data. At a $\mathrm{RH}$ of $10 \%$, the meniscus width gradually shrank from 37 to $17 \AA$ (drawn as circles) with increasing $d$ from 5 to $13 \AA$. With further increases in $d$, the meniscus snaps off to give a zero width. The fluctuation in the meniscus width ( $d_{\mathrm{s}}$, drawn as an error bar) was particularly large at a $d$ value just below the snap-off distance, indicating the instability of the meniscus. In this study, $d_{s} / 2 r_{2}$ was defined as the degree of instability. $d_{\mathrm{s}} / 2 r_{2}$ ranged from $5 \%$ to $32 \%$ for a RH of $10 \%$. Previously, menisci with $d_{s} / 2 r_{2}$ above $10 \%$ were defined as unstable ones. ${ }^{26}$ The meniscus width versus $d$ for RH of $40 \%$ (drawn as squares) revealed the same qualitative behavior as found for $\mathrm{RH}$ of $10 \%$. On the other hand, the meniscus width was larger than the corresponding width at $\mathrm{RH}$ of $10 \%$, and the snap-off distance increased to $17 \AA$. Note the decrease rate of the width with increasing $d$, that is, the slope of the linear fit to the data in Figure 2e is smaller for a $\mathrm{RH}$ of $40 \%$. This rate quantifies how much the meniscus width shrinks as the tip retracts from the surface. Previously, this rate was called the susceptibility of the meniscus width. ${ }^{25}$ The meniscus width at a $\mathrm{RH}$ of $40 \%$ was more stable in width with regard to the tip retraction. In general, the susceptibility of the width decreases with increasing humidity. Later, this susceptibility was related to the humidity dependence of the pull-off force (see below).

The meniscus has its maximum height and minimum width at the tip-surface distance immediately before its snap-off (for example, at $d$ s of 13 and $16 \AA$ for RHs of $10 \%$ and $40 \%$, respectively). These maximum heights and minimum widths of the meniscus were checked for various RHs (5-70\%). Figure 3 shows that as $\mathrm{RH}$ increases from $5 \%$ to $70 \%$, the maximum meniscus height (drawn as circles) increases gradually from 11 to $20 \AA$. Several steps are noticeable in the height versus the

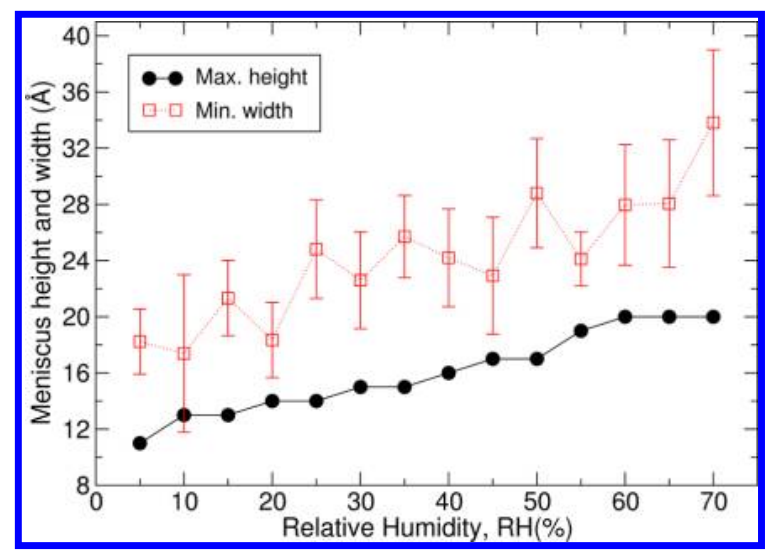

Figure 3. Maximum height and minimum width of the meniscus. The circles and squares are the height and the neck width of meniscus, respectively, just before its snap-off as the tip retracts from the surface. Lines are drawn as a visual guide, and the error bars represent the thickness of the meniscus periphery. 
$\mathrm{RH}$ curve, suggesting that an increase in $\mathrm{RH}$ sometimes does not alter the maximum height. On the other hand, the minimum width (Figure 3, drawn as squares) increases with small oscillations with increasing humidity. To understand the local decrease in the minimum width with increasing humidity, it should be noted that an increased height of the meniscus means a vertical stretching of the meniscus. Assuming the meniscus volume does not change, this vertical stretching should reduce the meniscus width. For example, as RH increases from $5 \%$ to $10 \%$ in Figure 3, the height increases from 11 to $13 \AA$. The increased height in turn stretches the meniscus vertically and decreases its width from 18.2 to $17.4 \AA$. This explanation strictly holds for the case where the volume of the meniscus is fixed. On the other hand, the meniscus volume increased with increasing $\mathrm{RH}$. Therefore, it is possible that an increased $\mathrm{RH}$ increases both the height and width of the meniscus. This is true for the transition of $\mathrm{RH}$ from $55 \%$ to $60 \%$. For all other cases, an increased height results in a decrease in width. If the height is unchanged with increasing $\mathrm{RH}$, such as in the case where $\mathrm{RH}$ varies from $10 \%$ to $15 \%$ in Figure 3, there is no vertical stretching of the meniscus due to an increase in height. The meniscus in this case increases in width with increasing humidity, as shown in Figure 3.

The minimum width shown in Figure 3 ranged from 17 to 33 $\AA$. If the molecular diameter of water is taken as $2.7 \AA{ }^{48}$ the minimum width corresponds to 6-12 molecules aligned laterally. Therefore, the narrowest meniscus in the current range of RHs is six molecules wide. Interestingly, this value is close to the estimate (five molecules wide) reported in previous lattice gas simulations. ${ }^{26}$ The degree of instability $d_{s} / 2 r_{2}$ ranged from $8 \%$ to $32 \%$. All the menisci except for a $\mathrm{RH}$ of $55 \%$ were unstable according to the previous criterion for the meniscus stability (below 10\%). ${ }^{26}$

The force exerted on the tip $F_{z}$ was investigated by varying $d$. Figure 4 plots $F_{z}$ versus $d$ for RHs of $10 \%$ (top) and $40 \%$ (bottom) (drawn as circles). $F_{z}$ was projected into contributions from the surface (van der Waals force) and the meniscus (capillary force). $F_{z}$ is negative (positive) if the tip feels an attraction (repulsion) from the meniscus and surface. The capillary force originating from the meniscus (drawn as triangles) governs the total force $F_{z}$, regardless of humidity, and is strongly repulsive at the shortest value of $d$. The attractive force from the surface (drawn as squares) is minor and serves as a small background to $F_{z}$ for distances greater than $8 \AA$. The capillary force is longer ranged than the surface force, approaching zero at a longer distance. By comparing the top and bottom of Figure 4, increasing the humidity makes the capillary force longer ranged: the capillary force reaches distances up to 14 and $18 \AA$ for RHs of $10 \%$ and $40 \%$, respectively. This range of force is longer than that of the force between silica or mica plates immersed in liquid water $(=12$ A). ${ }^{49}$

Interestingly, $F_{z}$ shows a damped oscillation with respect to $d: F_{z}$ is slightly repulsive at the shortest $d$ and reaches a minimum at $d=6 \AA$ at RHs of both $10 \%$ and $40 \%$ (see below for discussion on why the force is minimal at this $d$ value). This $d$ value at the minimal $F_{z}$ agrees with those found in the GCMC and the density functional theory studies adopting the lattice gas model. ${ }^{29,47}$ For RHs of both $10 \%$ and $40 \%, F_{z}$ increases to a local maximum at $d=8 \AA$ and then decreases with further increases in $d . F_{z}$ again increases starting at $d=10 \AA$. The force for a $\mathrm{RH}$ of $10 \%$ then approaches zero, but for a $\mathrm{RH}$ of $40 \%$, the force shows another maximum and minimum at $d=12$ and

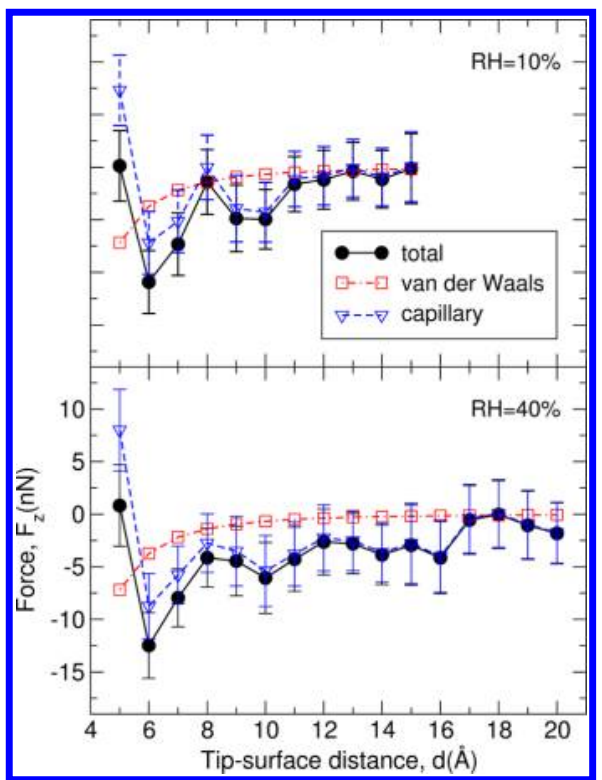

Figure 4. Force-distance curve. The $z$ component of the total force on the tip, $F_{z}$, vs the tip-surface separation $d$ is drawn as filled circles for $\mathrm{RHs}$ of $10 \%$ (top) and $40 \%$ (bottom). $F_{z}$ is projected into the contributions from the tip-surface (van der Waals force, drawn as squares) and tip-water (capillary force, drawn as triangles) interactions. A negative (positive) force represents an attractive (repulsive) force. Lines are drawn as a visual guide, and the error bars represent the standard deviation of the force.

$16 \AA$, respectively. As $d$ increases, the amplitude of the oscillation decreases. This oscillation in the capillary force was not captured by continuum theory ${ }^{21,24,50}$ or by the lattice model simulation. ${ }^{27,47}$ The peak-to-peak distance for $F_{z}(d)$ was approximately $3 \AA$, which is close to the molecular diameter of water. ${ }^{35}$ The oscillatory force was therefore attributed to the formation and destruction of water layers between the tip and surface. The force reaches a minimum when the $d$ value enables the complete layering of water. Otherwise, such a complete layering is broken, and the resulting structural instability of the meniscus gives an increased force. Consequently, the force shows alternating minima and maxima with increasing $d$. The present oscillation in the force qualitatively agrees with the previous experimental force data ${ }^{51-53}$ and simulation results. $^{54-56}$ For example, the experimental force between a silicon tip and mica surface exhibited oscillatory behavior with a peak-to-peak distance of $2-3 \AA^{53}$ The same type of oscillation was observed in the MD simulations on a mica slit ${ }^{54}$ and a carbon nanotube interacting with an alumina surface in solution. ${ }^{55}$ An oscillation in the force was also reported in the $\mathrm{MD}$ simulation on the indentation of a water-covered $\mathrm{MgO}$ surface with a probe tip. ${ }^{56}$

The atomic charge density averaged over $r, \rho_{\mathrm{e}}(h)$ was examined to gain insight on the water layering responsible for the force oscillations. Figure 5 presents a plot of $\rho_{\mathrm{e}}(h)$ for $d$ values ranging from 5 to $10 \AA$ (drawn as solid lines). Note hydrogen (oxygen) atoms give rise to positive (negative) charges. For comparison, the charge density was plotted for a slit system, where liquid water is confined between two parallel gold (111) plates (drawn as broken lines). Irrespective of $d$, two negative dips exist near the surface $(h=0)$ and tip $(h=d)$. The dip near the surface was deeper because it comes from the first layer that covers the entire gold surface. The other dip is relatively shallow because it arises from a layer above the first 


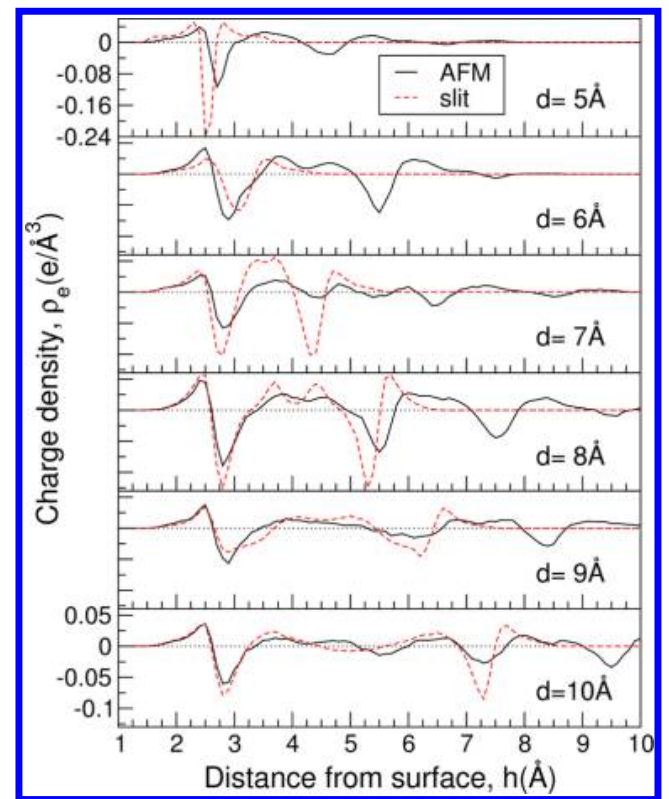

Figure 5. Atomic charge density as a function of the height from the surface. The charge density $\rho_{\mathrm{e}}$ is plotted as a function of the height $h$ for different tip-surface separations, $d s$. The axis scales drawn in the lowest panel apply to all panels except the top panel, where the $y$ axis was rescaled for better visualization.

layer, which does not extend laterally over the entire surface (see Figure 1). The $\rho_{\mathrm{e}}(h)$ of the present AFM system has dips, whose locations and depths are quite different from those of the slit system. The charge density of the slit system is approximately symmetric with respect to the $h=d / 2$ line and only exists for $h$ values less than the plate-to-plate distance $d$. The dips of the slit system are deeper because the confined water extends laterally over the entire slit. The charge density for the present AFM system exists for $h$ values larger than $d$ due to the spherical curvature of the tip (note $d$ is the distance of the tip end from the surface top). Owing to the tip curvature, the outer part of the meniscus can have incomplete layering, even though the inner part of the meniscus has well-developed layering and vice versa. At $d=6 \AA$, where the force is minimal in Figure 3, the two dips of $\rho_{\mathrm{e}}(h)$ are located at $h=2.8$ and 5.5 $\AA$, respectively. These dips are precisely one molecular diameter away from each other $(2.7 \AA)$. Therefore, there are two welldefined layers of water sandwiched between the tip and surface, which in turn gives the minimum $F_{z}$ (the strongest attraction between the tip and surface). The second layer must be off the tip axis $(r>0)$ because, with this $d$ value, the two layers cannot fit in along the tip axis (note there is only one dip for the slit system at the same value of $d$ ). At $d=7 \AA$, two shallow dips are located at $h=4.5$ and $5.4 \AA$. Their distances from each other and from the neighboring dips are well below the molecular diameter. Therefore, the layers are not fully developed, except for those near the surface and tip. At the same $d$ value, the slit system has two distinct dips almost equal in magnitude. Here, the dip-to-dip distance was well below (1.6 ̊) the molecular diameter of water, indicating that the layering is incomplete. At $d=8 \AA$, where the force is maximal for the present AFM system, there are three well-developed dips. Note the third dip is less than one molecular diameter $(2.0 \AA)$ away from the second dip, whereas the second layer is precisely one molecular diameter away from the first. Presumably, the third layer incommensurate with the second layer is responsible for the maximal force observed in Figure 3. This means that the water molecules in the third layer tend to increase their distances from the second layer from the current incommensurate value (2.0 ̊) to the molecular diameter to achieve complete layering. This will promote an increase in the tip-surface distance and therefore give the maximal force. On the other hand, the two dips separated by $2.7 \AA$ for the slit system at the same $d$ value represent complete layering. At $d=9 \AA$, the present AFM system shows almost three layers, considering that the dip-todip distances (3.2 and $2.3 \AA$ ) are close to the molecular diameter. Only two layers were observed for the slit system at the same $d$ value. Considering that the dip-to-dip distance is larger than the molecular diameter $(3.2 \AA)$, the layering is not as dense and complete as in the slit system with $d=8 \AA$. At the largest $d(=10 \AA)$, four dips exist for the present AFM system, and two dips in the middle are closer to each other $(1.8 \AA)$, meaning four layers are not yet fully formed. For the slit system with the same $d$ value, the distances between adjacent dips are smaller than the molecular diameter ( 2.2 and $2.3 \AA$ ), and the middle dip is very shallow. Therefore, the layer in the middle is not fully developed.

Figure 5 also provides information on the orientation of the water molecules. Regardless of $d$, there is a distinct positive peak near $2.5 \AA$ followed immediately by a negative dip at approximately $2.7 \AA$. Because the dip is only $0.2 \AA$ away from the peak, it is reasonable to assume they come from the same molecule. Therefore, a molecule in the first layer is believed to prefer the orientation where hydrogen atoms are closer to the surface. This preference for the hydrogen-down orientation of water molecules has been found in MD simulations of a carbon nanotube tip immersed in water, ${ }^{55}$ of a water film on a silica surface $^{57}$ and of water confined in nanoporous silica. ${ }^{58}$ This was attributed to the hydrogen-down configuration being energetically more stable than any other orientation of molecule. ${ }^{59,60}$ Similarly, a relatively small positive peak follows a negative dip located near $h=d$ (near the tip). This suggests that water molecules near the tip orient preferentially so that the hydrogen atoms are closer to the tip (here, the hydrogen up orientation is preferred). Owing to the spherical curvature of the tip, this preferential orientation is not as distinct as for the first layer next to the surface (the dip-to-peak transition near $h$ $=d$ is not as sharp as the peak-to-dip transition for the first layer). No preferential orientation of the molecule was observed in the intermediate layers between the first layer and the layer attached to the tip (for $h$ values above $2.5 \AA$ and below $d$ ).

Figure 6 shows how the meniscus is affected by humidity. Shown are the density profiles taken at RHs of $10 \%$ (a), $20 \%$ (b), $30 \%$ (c), and $40 \%$ (d) for a fixed tip-surface separation of $14 \AA$. At the lowest humidity (Figure 6a), water molecules were adsorbed separately on the tip and surface without forming a meniscus. As the humidity is increased to $20 \%, 30 \%$, and $40 \%$ (Figure $6 \mathrm{~b}-\mathrm{d}$ ), a bridging water meniscus forms and increases its width. By using the fitting eq 5 for the density profile, the meniscus neck widths $\left(2 r_{2} \mathrm{~s}\right)$ obtained were 18.6 (b), 24.4 (c), and $28.6 \AA$ (d). The periphery thickness of the meniscus $d_{\mathrm{s}}$ was 2.2 (b), 5.9 (c), and $7.2 \AA$ (d). The force on the tip, $F_{z}$, drawn as circles in Figure 6e, was calculated by varying $\mathrm{RH}$ from $10 \%$ to $60 \%$. The standard deviation of $F_{z}$ is drawn as an error bar. The degree of instability $d_{s} / 2 r_{2}$ varies as $12 \%, 24 \%, 25 \%, 26 \%$, and $24 \%$ for $\mathrm{RHs}$ of $20 \%, 30 \%, 40 \%, 50 \%$, and $60 \%$, respectively. As the humidity increases, the force becomes increasingly attractive. Therefore, with increasing humidity, the 


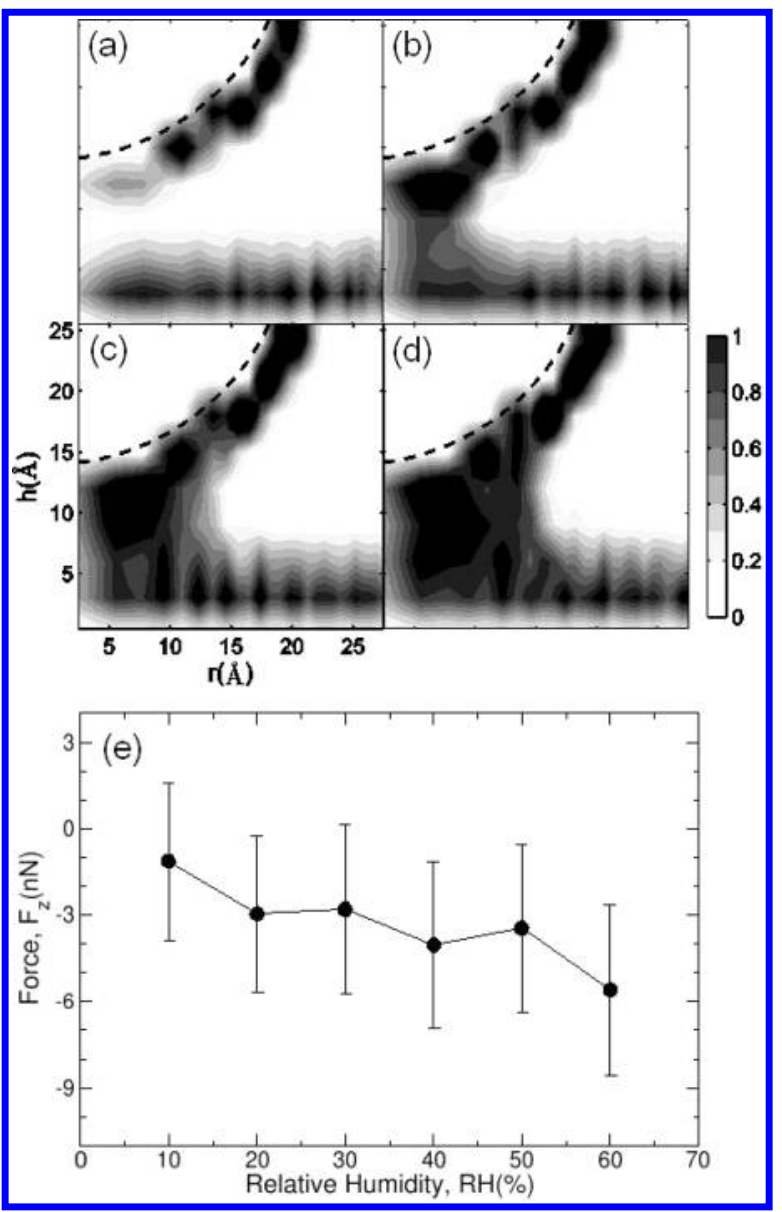

Figure 6. $(a-d)$ Humidity effects on the water meniscus. The density profile is plotted for a relative humidity of $10 \%$ (a), $20 \%$ (b), $30 \%$ (c), and $40 \%$ (d). In each panel, the tip boundary is drawn as a dashed line, and the surface is located at $h=0$. The axis scales drawn in (c) apply to (a), (b), and (d). (e) Humidity effects on the force exerted on the tip. At a fixed tip-surface separation of $14 \AA$, the force on the tip $F_{z}$ was calculated by varying RH from $10 \%$ to $60 \%$. The error bars are the standard deviations, and the line is drawn as a visual guide.

meniscus increases its size, and the magnitude of the capillary force increases. This monotonous increase in the magnitude of the force with increasing humidity was attributed to the force being calculated by fixing the tip-surface separation and varying the humidity only. Note that the force can also change by varying the tip-surface separation. Consequently, the maximum magnitude of the capillary force for a given humidity, so-called the pull-off force, is not necessarily a monotonically increasing function of $\mathrm{RH}$ (see below).

Experimentally, the pull-off force is defined as the force necessary to detach an AFM tip contacting with a surface. The pull-off force on a nanoscale AFM tip had a strong humidity dependence, in contrast to those on the tips with radii above 1 $\mu \mathrm{m} .{ }^{61}$ The force-distance curve $F_{z}(d)$ for a given humidity was obtained to calculate the pull-off force in the simulation, as shown in Figure 4. The pull-off force was then identified as the depth of the most attractive force in the $F_{z}(d)$ curve. The top of Figure 7 plots the pull-off force as a function of $\mathrm{RH}$. The pulloff force was $11.48 \mathrm{nN}$ on average, which is comparable to the experimental values. ${ }^{13-16,62}$ The pull-off force increased with increasing humidity from $5 \%$ to $20 \%$ and then decreased with further increases in humidity. The pull-off force reached a plateau above $30 \%$ humidity. This crossover humidity depend-

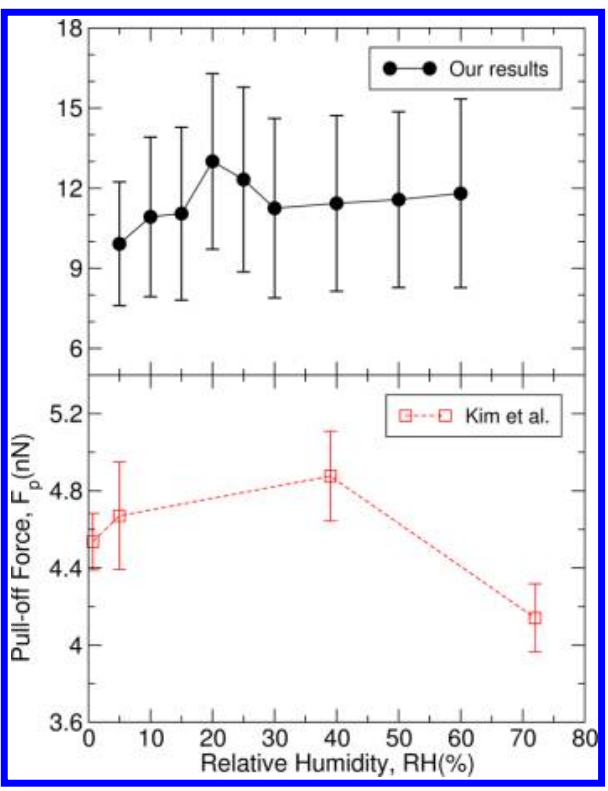

Figure 7. Humidity dependence of the pull-off force. By varying the tip-surface separation, the maximal attractive force was calculated for a given humidity. The magnitude of the maximal force is defined as the pull-off force at that RH. (Top) The pull-off force was plotted as a function of RH. Drawn as error bars are the standard deviations of the pull-off forces. (Bottom) The experimental pull-off force on a flat gold surface reported by Kim et al. ${ }^{33}$ In both panels, the line serves as a visual guide.

ence of the pull-off force agrees with that reported by Kim et al. ${ }^{33}$ They measured the pull-off force on a silicon nitride tip interacting with an atomically smooth gold surface, which is close to the present simulation system. As shown in the bottom of Figure 7, their experiment also shows a maximum in the pulloff force versus humidity curve. The maximum pull-off force in this simulation was located near $20 \% \mathrm{RH}$, but the force reported by Kim et al. ${ }^{33}$ was at $40 \%$. However, the experimental pull-off force is reported for RHs of $1 \%, 5 \%, 40 \%$, and $72 \%$ only. Therefore, the true maximum probably lies somewhere between $5 \%$ and $40 \% \mathrm{RH}$. The pull-off force of this simulation is 2 or 3 times larger than the experimental force. Presumably, the present gold tip is more hydrophilic than the silicon nitride tip used in their experiment, giving rise to a stronger pull-off force. Incidentally, the present $\mathrm{RH}$ for the maximum pull-off force coincides with that reported in an AFM experiment on a mica surface. ${ }^{16}$

The crossover (increasing and then decreasing) humidity dependence is typical for the pull-off force on a hydrophilic tip contacting with a hydrophilic surface. ${ }^{13,16,17,25,62-64}$ Sirghi $^{65}$ reported that a hydrophilic tip gives a maximum in the pull-off force near $30 \% \mathrm{RH}$, whereas the pull-off force for a hydrophobic tip increases monotonically. Similarly, Xiao and Qian ${ }^{18}$ reported that a hydrophilic surface gives a maximum pull-off force while the pull-off force on a hydrophobic surface is almost independent of the humidity. Jang et al. ${ }^{47}$ reported that the pull-off force is a constant, a monotonically increasing, and a nonmonotonic function of humidity for a hydrophobic, a weakly hydrophilic, and a strongly hydrophilic surface, respectively.

The humidity dependence of the pull-off force is often related to the tip shape. Pakarinen et al. showed theoretically that a drastically different humidity dependence, from a monotonic increase to a crossover behavior, results by simply 
changing the tip shape. ${ }^{61}$ As the tip curvature becomes more blunt (from spherical to flat bottomed), the humidity dependence changed from monotonic to crossover behavior. Farshchi-Tabrizi et al. reported a similar dependence on the tip shape by considering spherical, cone-shaped, and cylindrical tips in their continuum theory study. ${ }^{66}$ Similarly, Köber et al. reported that an almost flat nanometer-sized apex of the tip gives rise to a monotonous decrease in the pull-off force with increasing $\mathrm{RH}^{67}{ }^{67}$ Butt et al. ${ }^{68}$ reported that a tip shape can be inferred from the humidity dependence of the pull-off force in conjunction with a continuum theory (but the procedure was too complicated to be used routinely). In the present simulation, the tip shape was a cylinder capped with a hemisphere. At low humidity, the meniscus touched the hemispherical part of the tip only. As the humidity increased, the meniscus began to crawl over the cylinder wall. This is where the pull-off force began to decrease with increasing humidity. Farshchi-Tabrizi et al. also reported a change in the humidity dependence of the pull-off force as the meniscus starts to cover a different geometrical region of the tip. ${ }^{66}$

Efforts have been made to explain the humidity dependence of the pull-off force in terms of the molecular structure of the meniscus. Asay and Kim reported that the crossover (increasing and then decreasing) humidity dependence of the pull-off force could be reproduced by considering the icelike layers adsorbed on silica. ${ }^{17}$ Their spectroscopic data showed that the meniscus structure is mostly icelike at low humidity and liquid layers subsequently accumulate on top of the icelike structure with increasing humidity. After including the force due to the icelike structure, they could reproduce the experimental pull-off force versus humidity. No evidence of the presence of an icelike structure was found in the present simulation however, except for the partial order in the molecular orientation near the surface and tip. Jang et al. ${ }^{25}$ reported that the humidity dependence can be related to the structural susceptibility of the meniscus with regards to the retraction of the tip from the surface. They showed that the humidity dependence of the force is determined by the change in meniscus volume with increasing tip-surface distance. An increase in the tip-surface distance increases the height of the meniscus, increasing its volume. However, the same increase in the tip-surface distance reduces the meniscus width and meniscus volume. These two opposite contributions compete to determine if the pull-off force increases or decreases with increasing humidity. The susceptibility of the meniscus width to tip retraction emerges as a deciding factor. For strongly hydrophilic tips at high humidity, the water meniscus becomes wide and has low susceptibility (a small slope of the width vs distance curve shown in Figure 2e), so the meniscus volume expands as the tip is pulled away from the surface. When such a case arises, the pull-off force becomes a decreasing function of humidity.

Finally, there is inherent difficulty in determining the humidity dependence of the pull-off force both theoretically and experimentally. First, it is difficult to determine the precise geometry of a nanoscale tip experimentally. Moreover, there are possible structural changes in the tip due to the tip-surface interaction. Consequently, a repeated measurement under the same conditions will not reproduce the previous pull-off force measurement. ${ }^{66}$ In addition, different research groups reported different humidity dependences of the pull-off forces for the same tip and surface combination. ${ }^{13} \mathrm{~A}$ previous simulation showed that even a small difference in the tip shape (naturally introduced by an atomic scale roughness on the tip) can lead to a drastic difference in the humidity dependence of the pull-off force. ${ }^{27}$ Even if the tip geometry is known precisely and the tip does not suffer from deformation, the present simulation revealed large fluctuations in the pull-off force, as shown in the error bars in Figure 7.

\section{CONCLUSION}

GCMC simulations were performed using a realistic molecular model to examine the water meniscus condensed between a hydrophilic AFM tip and flat gold surface. The meniscus structure and capillary force due to the meniscus were investigated by varying both the humidity and tip-surface separation systematically. As the tip with nanometer asperity retracts from the surface, the meniscus shrinks at its neck and then snaps off. The fluctuation in the periphery of the meniscus increases drastically near the snap-off. The minimum width of the meniscus was found to be six molecules wide, which is in reasonable agreement with the previous estimate using the lattice gas model. The capillary force due to the meniscus dominates over the van der Waals force from the surface, and the force showed a damped oscillation with increasing tipsurface separation. This oscillation was attributed to the formation and destruction of the layering of water sandwiched between the tip and surface. The nanometer asperity of the AFM tip gives rise to a layering of water different from the corresponding layering of water sandwiched between two parallel plates. At a fixed tip-surface separation, the attractive force on the tip increased monotonously with increasing humidity. However, the pull-off force showed crossover behavior: an increase with increasing humidity from $5 \%$ to $20 \%$ and a decrease with further increases in humidity. This behavior agrees with the AFM experiment on a gold surface.

\section{AUTHOR INFORMATION}

\section{Corresponding Author}

*E-mail: jkjang@pusan.ac.kr.

\section{Notes}

The authors declare no competing financial interest.

\section{ACKNOWLEDGMENTS}

This study was supported by National Research Foundation Grants funded by the Korean Government (MEST) (nos. 2011-0003078 and 2011-0027696). J.J. wishes to thank the Korea Institute of Science and Technology Information for the use of the PLSI supercomputing resources. We thank Dr. DooIn Kim for providing the experimental data shown in Figure 7.

\section{REFERENCES}

(1) Basnar, B.; Willner, I. Small 2009, 5, 28-44.

(2) Manandhar, P.; Jang, J.; Schatz, G. C.; Ratner, M. A.; Hong, S. Phys. Rev. Lett. 2003, 90, 115505.

(3) Schenk, M.; Futing, M.; Reichelt, R. J. Appl. Phys. 1998, 84, 4880-4884.

(4) Jinesh, K. B.; Frenken, J. W. M. Phys. Rev. Lett. 2006, 96, 166103.

(5) Gelb, L. D.; Gubbins, K. E.; Radhakrishnan, R.; SliwinskaBartkowiak, M. Rep. Prog. Phys. 1999, 62, 1573-1659.

(6) Wang, J.; Qian, J.; Gao, H. Langmuir 2009, 25, 11727-11731.

(7) Piner, R. D.; Zhu, J.; Xu, F.; Hong, S.; Mirkin, C. A. Science 1999, 283, 661-663.

(8) Mirkin, C. A. ACS Nano 2007, 1, 79-83.

(9) Weeks, B. L.; Vaughn, M. W.; DeYoreo, J. J. Langmuir 2005, 21, 8096-8098.

(10) Weeks, B. L.; DeYoreo, J. J. J. Phys. Chem. B 2006, 110, 1023110233. 
(11) Choe, H.; Hong, M.-H.; Seo, Y.; Lee, K.; Kim, G.; Cho, Y.; Ihm, J.; Jhe, W. Phys. Rev. Lett. 2005, 95, 187801.

(12) Lee, M.; Sung, B.; Hashemi, N.; Jhe, W. Faraday Discuss. 2009, 141, 415-421.

(13) Sedin, D. L.; Rowlen, K. L. Anal. Chem. 2000, 72, 2183-2189.

(14) Jones, R; Pollock, H. M.; Cleaver, J. A. S.; Hodges, C. S. Langmuir 2002, 18, 8045-8055.

(15) Chen, L.; Gu, X.; Fasolka, M. J.; Martin, J. W.; Nguyen, T. Langmuir 2009, 25, 3494-3503.

(16) Xu, L.; Lio, A.; Hu, J.; Ogletree, D. F.; Salmeron, M. J. Phys. Chem. B 1998, 102, 540-548.

(17) Asay, D. B.; Kim, S. H. J. Chem. Phys. 2006, 124, 174712.

(18) Xiao, X.; Qian, L. Langmuir 2000, 16, 8153-8158.

(19) Gao, C. Appl. Phys. Lett. 1997, 71, 1801-1803.

(20) Fei, K.; Chiu, C. P.; Hong, C. W. Microfluid. Nanofluid. 2008, 4, 321-330.

(21) Choi, H. J.; Kim, J. Y.; Hong, S. D.; Ha, M. Y.; Jang, J. Mol. Simul. 2009, 35, 466-472.

(22) Kim, H.; Saha, L. C.; Saha, J. K.; Jang, J. Scanning 2010, 32, $2-8$.

(23) Ko, J.-A.; Choi, H.-J.; Ha, M.-Y.; Hong, S.-D.; Yoon, H.-S. Langmuir 2010, 26, 9728-9735.

(24) Butt, H.-J.; Kappl, M. Adv. Colloid Interface Sci. 2009, 146, 4860.

(25) Jang, J.; Yang, M.; Schatz, G. J. Chem. Phys. 2007, 126, 174705.

(26) Jang, J.; Schatz, G. C.; Ratner, M. A. Phys. Rev. Lett. 2004, 92, 085504 .

(27) Jang, J.; Ratner, M. A.; Schatz, G. C. J. Phys. Chem. B 2005, 110, 659-662.

(28) Paramonov, P. B.; Lyuksyutov, S. F. J. Chem. Phys. 2005, 123, 084705 .

(29) Men, Y.; Zhang, X.; Wang, W. J. Chem. Phys. 2009, 131, 184702.

(30) Wynveen, A.; Bresme, F. J. Chem. Phys. 2006, 124, 104502.

(31) Dzubiella, J.; Hansen, J.-P. J. Chem. Phys. 2004, 121, 5514-5530.

(32) Giovambattista, N.; Debenedetti, P. G.; Rossky, P. J. J. Phys.

Chem. B 2007, 111, 9581-9587.

(33) Kim, D.-I.; Grobelny, J.; Pradeep, N.; Cook, R. F. Langmuir 2008, 24, 1873-1877.

(34) Ahn, Y.; Hong, S.; Jang, J. J. Phys. Chem. B 2006, 110, 42704273.

(35) Berendsen, H. J. C.; Postma, J. P. M.; van Gunsteren, W. F.; Hermans, J. Intermolecular Forces; Pullman, B., Ed.; Reidel Publishing Company: Dordrecht, The Netherlands, 1981.

(36) Allen, M. P.; Tildesley, D. J. Computer Simulation of Liquids; Claredon Press: Oxford, U.K., 1987.

(37) Heinz, H.; Vaia, R. A.; Farmer, B. L.; Naik, R. R. J. Phys. Chem. C 2008, 112, 17281-17290.

(38) Peng, D.-Y.; Robinson, D. B. Ind. Eng. Chem. Fundam. 1976, 15, $59-64$.

(39) Willems, H. H.; Poulis, J. A.; Massen, C. H. Thermochim. Acta 1986, 103, 137-145.

(40) Frenkel, D.; Smit, B. Understanding Molecular Simulation: From Algorithms to Applications (Computational Science), 2nd ed.; Academic Press: San Diego, CA, 2002.

(41) Liu, B.; Smit, B.; Calero, S. J. Phys. Chem. B 2006, 110, 2016620171.

(42) Dubbeldam, D.; Calero, S.; Vlugt, T. J. H.; Krishna, R.; Maesen, T. L. M.; Smit, B. J. Phys. Chem. B 2004, 108, 12301-12313.

(43) Werder, T.; Walther, J. H.; Jaffe, R. L.; Halicioglu, T.; Koumoutsakos, P. J. Phys. Chem. B 2003, 107, 1345-1352.

(44) Marquardt, D. J. Soc. Ind. Appl. Math. 1963, 11, 431-441.

(45) Berendsen, H. J. C.; Postma, J. P. M.; van Gunsteren, W. F.; DiNola, A.; Haak, J. R. J. Chem. Phys. 1984, 81, 3684-3690.

(46) Smith, W.; Yong, C. W.; Rodger, P. M. Mol. Simul. 2002, 28, 385-471.

(47) Jang, J.; Schatz, G. C.; Ratner, M. A. J. Chem. Phys. 2004, 120, $1157-1160$

(48) Schatzberg, P. J. Phys. Chem. 1967, 71, 4569-4570.

(49) Malani, A.; Ayappa, K. G.; Murad, S. J. Phys. Chem. B 2009, 113, $13825-13839$.
(50) Stifter, T.; Marti, O.; Bhushan, B. Phys. Rev. B 2000, 62, 1366713673.

(51) Singh, S.; Houston, J.; van Swol, F.; Brinker, C. J. Nature 2006, $442,526$.

(52) Fukuma, T.; Higgins, M. J.; Jarvis, S. P. Biophys. J. 2007, 92, 3603-3609.

(53) Fukuma, T.; Ueda, Y.; Yoshioka, S.; Asakawa, H. Phys. Rev. Lett. 2010, 104, 016101.

(54) Das, S. K.; Sharma, M. M.; Schechter, R. S. J. Phys. Chem. 1996, 100, 7122-7129.

(55) Argyris, D.; Ashby, P. D.; Striolo, A. ACS Nano 2011, 5, 22152223.

(56) Kendall, K.; Dhir, A.; Yong, C. W. Philos. Mag. 2010, 90, 41174128.

(57) Argyris, D.; Tummala, N. R.; Striolo, A.; Cole, D. R. J. Phys. Chem. C 2008, 112, 13587-13599.

(58) Bonnaud, P. A.; Coasne, B.; Pellenq, R. J.-M. J. Phys.: Condens. Matter 2010, 22, 284110.

(59) Roudgar, A.; Groß, A. Chem. Phys. Lett. 2005, 409, 157-162.

(60) Vassilev, P.; van Santen, R. A.; Koper, M. T. M. J. Chem. Phys. 2005, 122, 054701.

(61) Pakarinen, O. H.; Foster, A. S.; Paajanen, M.; Kalinainen, T.; Katainen, J.; Makkonen, I.; Lahtinen, J.; Nieminen, R. M. Modell. Simul. Mater. Sci. Eng. 2005, 13, 1175-1186.

(62) Wang, L.-F.; Rong, W.-B.; Sun, L.-N.; Chen, L.-G.; Shao, B. Chin. Phys. Lett. 2009, 26, 126801.

(63) He, M.; Blum, A. S.; Aston, D. E.; Buenviaje, C.; Overney, R. M.; Luginbühl, R. J. Chem. Phys. 2001, 114, 1355-1360.

(64) Huang, C.-C.; Chen, L.; Gu, X.; Zhao, M.; Nguyen, T.; Lee, S. J. Mater. Res. 2010, 25, 556-562.

(65) Sirghi, L. Appl. Phys. Lett. 2003, 82, 3755-3757.

(66) Farshchi-Tabrizi, M.; Kappl, M.; Cheng, Y.; Gutmann, J.; Butt, H.-J. Langmuir 2006, 22, 2171-2184.

(67) Köber, M.; Sahagún, E.; García-Mochales, P.; Briones, F.; Luna, M.; Sáenz, J. J. Small 2010, 6, 2725-2730.

(68) Butt, H.-J.; Farshchi-Tabrizi, M.; Kappl, M. J. Appl. Phys. 2006, $100,024312$. 